Published in "Physica A: Statistical Mechanics and its Applications 499: 490-497, 2018" which should be cited to refer to this work.

\title{
Collective iteration behavior for online social networks
}

\author{
Jian-Guo Liu ${ }^{\mathrm{a}, *}$, Ren-De Li ${ }^{\mathrm{b}, \mathrm{c}}$, Qiang Guo ${ }^{\mathrm{b}}$, Yi-Cheng Zhang ${ }^{\mathrm{d}}$ \\ ${ }^{a}$ Research Center of Fin. Tech. and Shanghai Key Lab. of Fin. Inf. Tech., Shanghai University of Finance and Economics, Shanghai 200433, \\ PR China \\ ${ }^{\mathrm{b}}$ Research Center of Complex Systems Science, University of Shanghai for Science and Technology, Shanghai 200093, PR China \\ c Library, University of Shanghai for Science and Technology, Shanghai 200093, PR China \\ ${ }^{d}$ Department of Physics, University of Fribourg, CH-1700 Fribourg, Switzerland
}

\section{H I G H L I G H T S}

- There are robust social signatures of interactions no matter how friend changes over time.

- The model shows that new users intend to look for the old users with strong ties while old users have tendency to interact with new friends.

- The presented model can reproduce the heterogeneity of social signature by adjusting the number of communicating targets and the max number of interactions.

Keywords: Social signature

Ego networks

Preferential interaction

\begin{abstract}
Understanding the patterns of collective behavior in online social network (OSNs) is critical to expanding the knowledge of human behavior and tie relationship. In this paper, we investigate a specific pattern called social signature in Facebook and Wiki users' online communication behaviors, capturing the distribution of frequency of interactions between different alters over time in the ego network. The empirical results show that there are robust social signatures of interactions no matter how friends change over time, which indicates that a stable commutation pattern exists in online communication. By comparing a random null model, we find the that commutation pattern is heterogeneous between ego and alters. Furthermore, in order to regenerate the pattern of the social signature, we present a preferential interaction model, which assumes that new users intend to look for the old users with strong ties while old users have tendency to interact with new friends. The experimental results show that the presented model can reproduce the heterogeneity of social signature by adjusting 2 parameters, the number of communicating targets $m$ and the max number of interactions $n$, for Facebook users, $m=n=5$, for Wiki users, $m=2$ and $n=8$. This work helps in deeply understanding the regularity of social signature.
\end{abstract}

\section{Introduction}

Collective behaviors of online users have been extensively investigated, which is of great significance for identifying the human communication patterns [1-3]. Oliveira et al. [4] found the scaling-law in Darwin's and Einstein's correspondence patterns. Brockman et al. [5] argued that the distribution of human traveling distances decayed as a power law. Saramäki [6] analyzed the mobile phone call pattern and found that human had persistence communication pattern. To explain the

\footnotetext{
* Corresponding author.

E-mail address: liujg004@ustc.edu.cn (J.-G. Liu).
} 


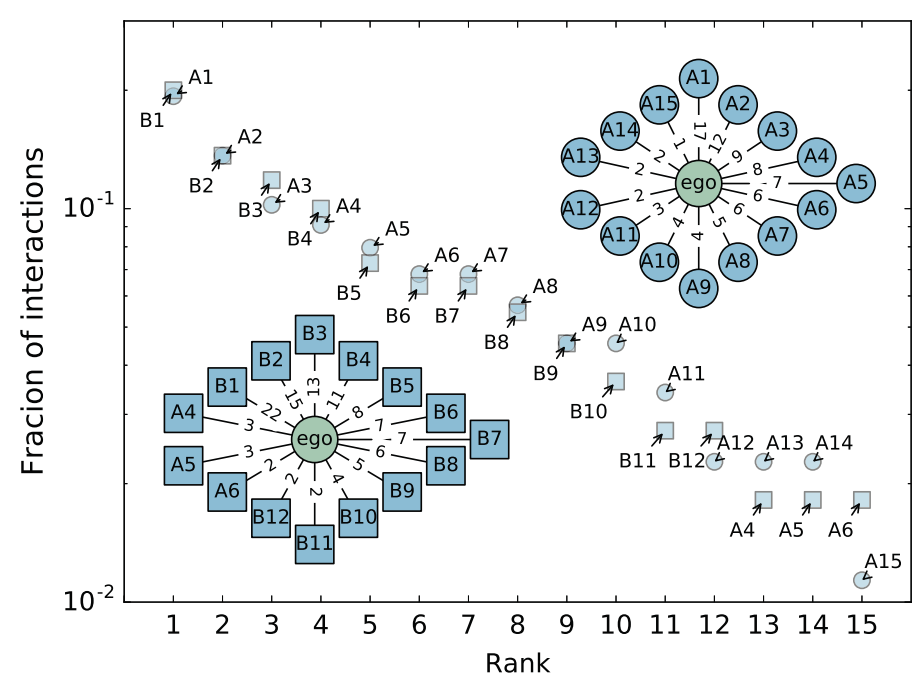

Fig. 1. Illustration of a typical users social signature. There are two consecutive time intervals 1 and 2, where the ego's alter naming A1-A15 and B1-B13 respectively. Meanwhile, in the interval 2, three alters A4, A5, A6 are the same alters occurred in the interval 1 . Top- 15 frequently contacted friends of the user are used to construct ego network base on the number of interactions. The turnover is defined by the Jaccard measurement, which is defined as the ratio of common alters over total alters in two intervals, in this case, $3 / 27=0.1111$. The two distractions represent two social signatures of the ego in each interval, where the social signature is defined as the fraction of interactions to the alter of each rank, which approximately follows the form of exponential distribution. Persistence of two social signatures is defined as the similarity between two probability distributions.

behavior patterns, the task- and interest-based models have been proposed. Task-based models [7-10] believed that human behaviors could be described as a decision-based process and tasks were executed according to their priorities. Interestdriven models [11-14] argued that the interest played an important role. Besides, circadian-driven models $[15,16]$ found that the day-night or weekly pattern existed in communication behaviors.

Among these collective behaviors, online social networks (OSNs) [17] got more and more attention. Hundreds millions users to communicate and interact with each other over the past years, which is broaden the knowledge about the human relation patterns [18-21]. Although it is convenient for users to build connection with more friends by OSNs, due to the cognitive ability $[22,23]$ and memory capacity $[24,25]$ constraints, the communication pattern of OSNs are found similar to the offline face-to-face networks [26]. Dunbar argued that, limited to human cognitive ability, average speaking, each individual could only maintain 150 friends, who has only 5 closest friends [27], which has been verified by face-to-face networks [28,29], mobile communication relationships [6,30], and online social networks [31-34].

Even in the limited scale of meaningful relations, by investigating the collective behaviors, there still are plenty measurements to find the statistical patterns of human interactions. From the respective of online social behavior, rating or comment behavior are commonly used to identify the human relations [35,36]. The network measurements are also widely used, such as tie strength which refers to the 'closeness' of a friendship in social ties; it captures a spectrum that ranges from strong ties with close friends to weak ties with more distant acquaintances [37-39], embeddedness which capture the number of mutual friends shared by its endpoints [40,39]. Moreover, the mechanisms of human interactions can be characterized by the weighted networks. Several models like the mutual selection model [41], generalized local-world models [42] provide the insights of the structure of interactions. Notably, different with the traditional statistical properties, social signature found in mobile phone [6] could be a general pattern which may be suitable for OSNs, but the statistical patterns and generation mechanism are still little known.

In this paper, we empirically investigate the pattern of social signature of the online Facebook and Wiki user. Social signature is constructed as illustrated in Fig. 1. By averaging the all participants who have the characteristic of social signature, we investigate the persistence of collective egos' social signatures and compare with a random null model. The empirical results show the heterogeneity of social signature. Then we present a model to reproduce the social signature and find that the number of communicating targets and the number of interactions could be two key factors of social signature.

\section{Empirical analysis}

\subsection{Materials}

The Facebook-wall $[43,44]$ (short for FW) and Wiki talk temporal $[45,46]$ (short for WT) datasets are investigated in this paper (see Table 1). In FW, a user can post comments on his(her) friends' walls, and these comments can be seen by visitors, while in the WT, interaction represents that a user edited another user's talk page. In order to make the time interval comparable, the FW spans from September 5, 2006 to December 31, 2008 and the WT spans from September 9, 
Table 1

Basic statistical properties of the Facebook-Wall (short for FW) and Wiki-Talk (short for WT) dataset, including the time span of each interval $i$ and the number of nodes $N$, edges $E$, common egos $c$ which is those who have social signatures in all 4 intervals.

\begin{tabular}{|c|c|c|c|c|}
\hline Interval & Time span & $N$ & E & $c$ \\
\hline & Facebook-Wall & & & \\
\hline$i=1$ & 2006-09-05-2007-04-05 & 10,836 & 106,624 & 77 \\
\hline$i=2$ & 2007-04-05-2007-11-03 & 16,844 & 196,035 & 77 \\
\hline$i=3$ & 2007-11-03-2008-06-02 & 21,960 & 185,803 & 77 \\
\hline \multirow[t]{2}{*}{$i=4$} & $2008-06-02-2008-12-31$ & 39,199 & 308,412 & 77 \\
\hline & Wiki-Talk & & & \\
\hline$i=1$ & 2005-09-09-2006-04-09 & 165,922 & 944,086 & 913 \\
\hline$i=2$ & 2006-04-09-2006-11-07 & 279,182 & $1,586,629$ & 913 \\
\hline$i=3$ & 2006-11-07-2007-06-07 & 455,810 & $2,317,985$ & 913 \\
\hline$i=4$ & 2007-06-07-2008-01-05 & 451,309 & $2,336,921$ & 913 \\
\hline
\end{tabular}

2005 to January 5, 2008. Datasets are separated into $I$ intervals evenly, here $I=4$, and each interval includes 212 days. Here, we treat interactions as indirect links since we only need to know the connection of ego and alters when calculating the social signature. The information of each interaction consists three parts: The wall/talk page owner (ego), the user who posted/edited (alter) and the corresponding posted/edited time. Then, in each interval, ego networks are constructed as the list of egos who has at least $k$ alters and take top- $R$ closest alters by counting interactions, here $k=15$.

In each interval, we construct ego networks, where egos and alters are tied to each other by online social relations. Tie strength is the number of interactions of each ego $j$ to alters in each interval $i$ and the alters are ranked on descending order by the number of interactions. Then, we calculate the social signature $P_{i, j}$ of ego $j$ in the interval $i$, defined by the fraction of interactions to the alter of each rank (Eqs. (1) and (2)). Here, only those who have at least 15 alters are taken into account to calculate social signatures. Meanwhile, we target the group of egos who appeared in all 4 intervals as collective egos. To quantify the similarity of social signatures, we use the Jensen-Shannon Divergence (short for JSD) to measure the distance of the social signatures between two egos. JSD $=0$ stands for the fact that two social signatures are identical. The smaller the $J S D$ value is, the more similar two social signatures are, which means fraction of interactions has persistence over time. We use Jaccard (short for $J$ ) to quantify the turnovers of two different sets of alters. $J=0$ represents that there are no common alters, while $J=1$ represents the exact same alters staying in consecutive intervals. As illustrated in Fig. 1, the number of common alters equals $30 \cdot \mathrm{J} /(1+J)$, in this case, $J=0.1111$, showing that there are 3 common alters between two consecutive intervals.

\subsection{Measurements}

Social signature $P$. The social signature of ego $j$ in the interval $i$, saying $P_{i, j}$, is defined as fraction of interactions of each alters after ranking and taking top $R$ alters (here $R=15$ ), which can be read as,

$$
P_{i, j}(X=r)=\frac{n_{i, j}(r)}{\sum_{r=1}^{R} n_{i, j}(r)}, r=1,2, \ldots, R
$$

where $X$ is discrete random variable representing the rank of alter, and $n_{i, j}(r)$ is the number of interactions given by an ego $j$ to the alter $r$ in the interval $i$. The rank $r$ ranges from 1 to $R$. Using Fig. 1 as a example, in intervals 1 , the number of interactions $n_{i, j}(r)$ are $\{17,12,9,8,7,6,6,5,4,4,3,2,2,2,1\}$, which is sorted by alters. Then, individual social signature $P_{1,1}$ is the frequency distribution, which is $\{0.1932,0.1364,0.1023,0.0909,0.0795,0.0682,0.0682,0.0568,0.0455,0.0455,0.0341$, $0.0227,0.0227,0.0227,0.0114\}$.

Meanwhile, to measure collective social signature in each interval $i$, we define the average social signature $\left\langle P_{i}\right\rangle$ as:

$$
\left\langle P_{i}\right\rangle=\frac{1}{c} \sum_{j=1}^{c} P_{i, j}(X=r), r=1,2, \ldots, R,
$$

where $\left\langle P_{i}\right\rangle$ is the average fraction of interactions to the alter of rank $r$ in the interval $i, i=1,2, \ldots, 4$. The social signature are averaged by the common egos appearing in 4 intervals, for FB users $c=77$, and for WT users $c=913$.

Jensen-Shannon Divergence JSD. Jensen-Shannon Divergence (short for JSD) [6] is introduced to measure the similarity between two different social signatures $P_{1}$ and $P_{2}$, defined as:

$$
\begin{aligned}
& J S D\left(P_{1}, P_{2}\right)=H\left[\frac{1}{2} P_{1}+\frac{1}{2} P_{2}\right]-\frac{1}{2}\left[H\left(P_{1}\right)+H\left(P_{2}\right)\right], \\
& H(P)=-\sum_{r=1}^{R} p(r) \log p(r),
\end{aligned}
$$


where $P_{1}$ and $P_{2}$ are two social signatures to be compared, which are the form of the distributions. If comparing collective egos' signatures between two consecutive time intervals, $P_{1}=\left\langle P_{i}\right\rangle$ and $P_{2}=\left\langle P_{i+1}\right\rangle$. If comparing collective egos' social signatures between empirical results and simulation result (saying $Q$ ) in the same interval, $P_{1}=\left\langle P_{i}\right\rangle$ and $P_{2}=\langle Q\rangle$. Additionally, $H(P)$ represents the Shannon entropy, where $p(r)$ refers to Eq. (2) if calculating JSD value of individual ego $j$, and refers to Eq. (4) if calculating JSD value of collective egos. From the definition of JSD, the lower bound is $J S D=0$ only when two distributions are identical. The smaller JSD value is, the more persistence of two social signatures are.

Jaccard $J$. We define $X_{i, j}$ as the set of alters, which contains 10 alters here, for ego $j$ in the interval $i$. The difference between two sets $X_{i, j}$ and $X_{i+1, j}$ of alters in two consecutive intervals $i$ and $i+1$, namely turnover, is measured by the Jaccard coefficient $J$,

$$
J\left(X_{i, j}, X_{i+1, j}\right)=\frac{\left|X_{i, j} \cap X_{i+1, j}\right|}{\left|X_{i, j} \cup X_{i+1, j}\right|} .
$$

The value of turnover $J$ lies in $[0,1] . J=1$ means that all alters of target ego are the same over time, and $J=0$ stands for no common alters. The low Jaccard value means that two sets of alters have little common alters, in another word, turnover of alters is high.

Then, we calculate the Jaccard averaged over collective egos in two consecutive intervals $i$ and $i+1$ :

$$
\left\langle J\left(X_{i, .}, X_{i+1,}\right)\right\rangle=\frac{1}{c} \sum_{j=1}^{c} J_{j}\left(X_{i, j}, X_{i+1, j}\right)
$$

where the number of common egos $c=77$ for FB dataset and $c=913$ for WT dataset.

Slope $\lambda$. The average social signature $\left\langle P_{i}\right\rangle$ is approximately the form of exponential distribution, as is shown in Fig. 2, we fit the distribution by the following probability density function:

$$
f(x)=\lambda \cdot e^{-\lambda x+a},
$$

where the parameter $\lambda$ is the slope of social signature and variable $x$ is the rank $1,2, \ldots, R$. The larger the parameter $\lambda$ is, the steeper the distribution is.

Null model. In order to examine that social signature deviates from randomness, a null model is introduced to compare with the empirical results, which is constructed as follows: (i) Randomly reshuffle alters, reconnecting the reshuffled alter to ego and remain timestamp, (ii) Construct the ego network based on reshuffled data, and then calculate the average social signature $\left\langle P_{i}\right\rangle$ according to Eq. (3).

\subsection{The empirical results}

\section{The persistence of social signature}

For the egos who have more than 15 alters in all 4 intervals, first, we calculate the average social signature $\left\langle P_{i}\right\rangle$ by averaging over the set of all egos' fraction of interactions in each internal $i$ (Eq. (2)). As shown in Fig. 2(a) and (c), the shape of average social signature $\left\langle P_{i}\right\rangle$ of collective egos in each interval exhibits the form of exponential distribution in both datasets (Fig. 2(a) and (c)). Then, we calculate the similarity of two average social signatures between two consecutive time intervals $J S D\left(\left\langle P_{i}\right\rangle,\left\langle P_{i+1}\right\rangle\right)(i=1,2,3,4)$ by Eq. (3). For the entire networks of participants, $J S D\left(\left\langle P_{1}\right\rangle,\left\langle P_{2}\right\rangle\right)=0.00024300$, $J S D\left(\left\langle P_{2}\right\rangle,\left\langle P_{3}\right\rangle\right)=0.00009842, J S D\left(\left\langle P_{3}\right\rangle,\left\langle P_{4}\right\rangle\right)=0.0008564$ for FW users and $J S D\left(\left\langle P_{1}\right\rangle,\left\langle P_{2}\right\rangle\right)=0.00107555, J S D\left(\left\langle P_{2}\right\rangle,\left\langle P_{3}\right\rangle\right)=$ $0.00006892, J S D\left(\left\langle P_{3}\right\rangle,\left\langle P_{4}\right\rangle\right)=0.00002275$, which suggest that the interactive frequency of ego to alters remain stable between two consecutive time intervals. Second, to measure the turnover of alters through time, we calculate the Jaccard of each long-term ego between two consecutive intervals $J\left(X_{i, j}, X_{i+1, j}\right)$ (Eq. (5)), where $X_{i, j}$ and $X_{i+1, j}$ are the two sets of the alters interacted with the ego $j$ in time interval $i$ and $i+1$ separately. The distributions of collective Jaccard in each interval are shown as violin plot in Fig. 2 (b) and (d), with the mean value calculated by Eq. (6). For the FW users, $J\left(X_{1, .}, X_{2, .}\right)=0.29 \pm 0.12$, $J\left(X_{2, .}, X_{3, .}\right)=0.27 \pm 0.13, J\left(X_{3, .}, X_{4, .}\right)=0.23 \pm 0.21$, which means that there are average 3-9 common alters over time. For the WT users, $J\left(X_{1, .}, X_{2, .}\right)=J\left(X_{2, .}, X_{3, .}\right)=J\left(X_{3, .}, X_{4, .}\right)=0.09 \pm 0.07$, which means there are average $1-4$ common alters over time. To sum up, whether turnovers of alters changing or stable, bigger or smaller, the average social signatures of long-term egos over time have highly persistence.

\section{The heterogeneity of social signature}

We introduce a null model to regenerate the data for comparing the frequency of interactions. The null model reshuffles all alters into random order and reallocate alters to each ego, so that the egos interactive patterns no longer exist. As shown in Fig. 2, collective signatures approximately follow exponential distributions. Then, we use the parameter $\lambda$ of exponential distribution to depict the shape of signature (Eq. (7)). For each distribution in Fig. 4, we use non-linear least squares to fit a function. Fig. 3 shows the value of slope $\lambda$ for empirical results and null model results for average social signatures of collective egos in each interval. There are 4 distributions, which represent 4 average social signatures in 4 time intervals (calculated according to Eq. (2)). One can find that the values of empirical $\lambda$ in the FW dataset are around 0.26 , and the values 

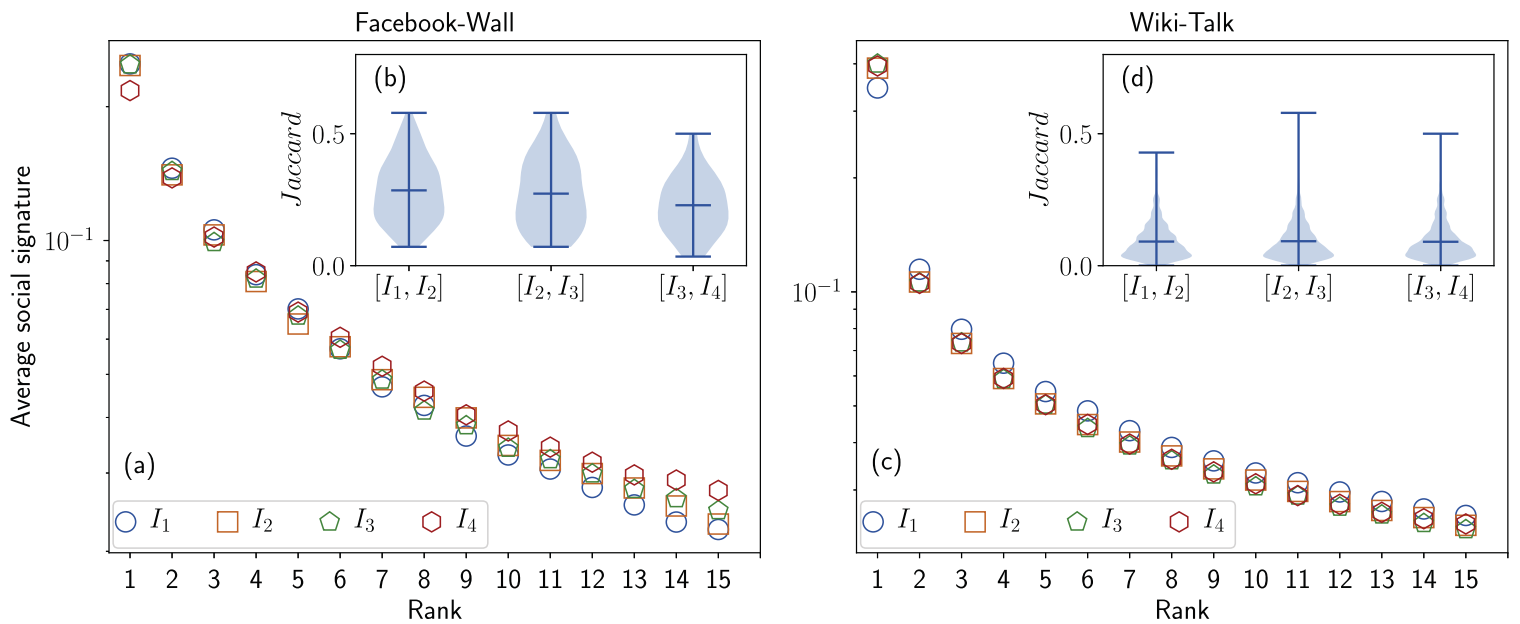

Fig. 2. The average social signature $\left\langle P_{i}\right\rangle$ and turnover $J\left(X_{i, j}, X_{i+1, j}\right)$ of collective egos for the FW and WT datasets. The shapes of average social signatures are invariant (seen in subplot (a) and (c) for the distributions in interval $I_{1}, I_{2}, I_{3}, I_{4}$ ), although alters display different turnovers as indicated by the Jaccard between two consecutive intervals $\left(\left[I_{i}, I_{i+1}\right](i=1,2, \ldots, 4)\right)$ for two datasets (seen in subplot (b) and (d) as violin plots). For the FW users, the average Jaccard in consecutive intervals are $0.29,0.28$, and 0.25 respectively. For the WT users, average Jaccard equals $0.10,0.09$ and 0.09 throughout time intervals.

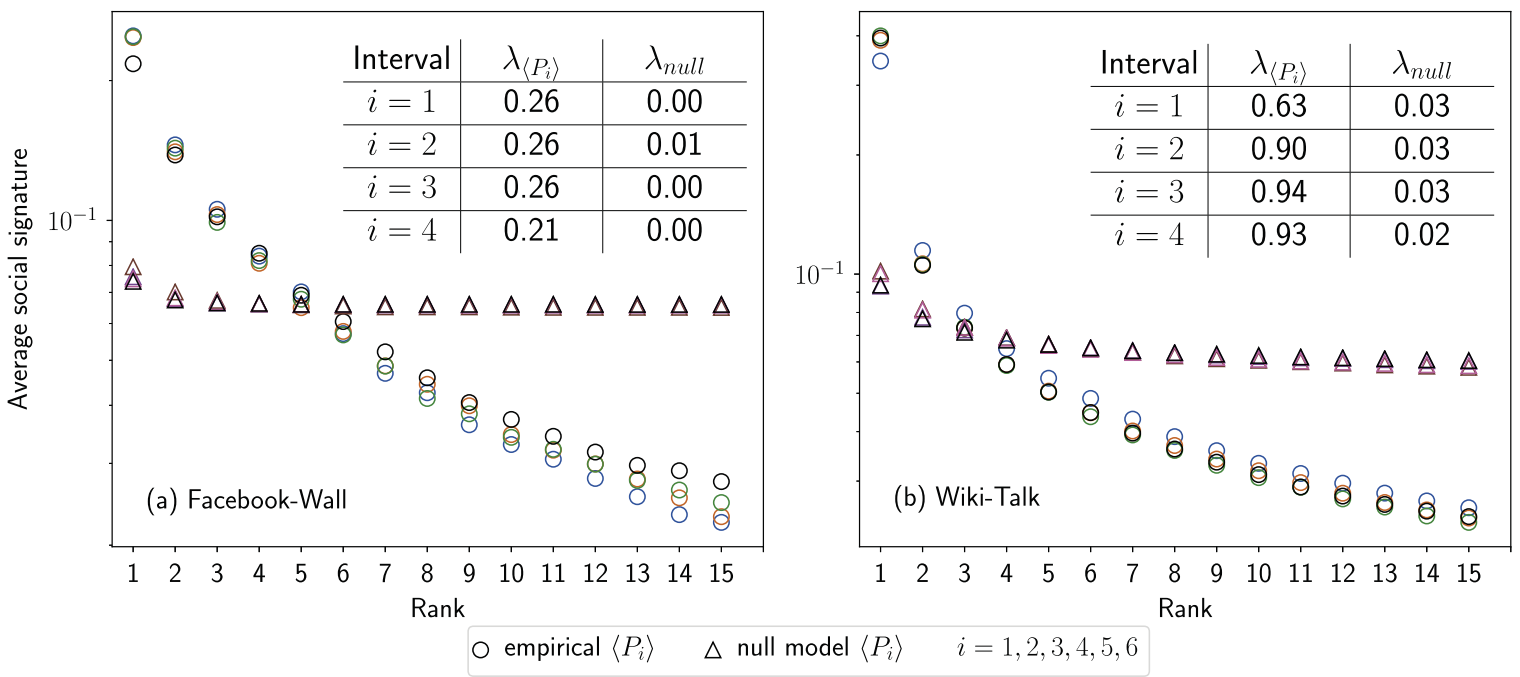

Fig. 3. Slope $\lambda$ is the parameter of exponential distribution used to depict the shape of average social signature of collective egos $\left\langle P_{i}\right\rangle$ denoted by $\lambda\left\langle P_{i}\right\rangle$ in each interval $i, i=1,2,3,4$, compared between empirical results and the null model. Each marker stands for 4 average social signatures ( 4 distributions) of each group of results. The comparisons show that average social signatures of empirical results are steeper than that of the null model, which indicate that social signature has heterogeneous characteristic.

of $\lambda$ in the WT dataset are round 0.90 , while the $\lambda$ values of each null model are all close to 0 , which shows that empirical social signatures have steeper slope. The results indicates that ego's tie strength with alters decreases with the frequency of interactions, much faster than the null model, namely, the social signature has heterogeneous characteristic.

\section{The model analysis}

\subsection{The model construction}

To regenerate collective behaviors of online social signature, we present a preferential interaction model in terms of weighted networks. Weighted networks are often described by a weighted adjacency matrix $w_{i j}$ which represents the weight on the edge connecting vertices $i$ and $j$, with $i, j=1, \ldots, N$, where $N$ is the size of the network. In this model, we assume that there are two mechanisms for communications, topological growth and preferential interaction. We initiate a network with $m$ nodes and no edges. 

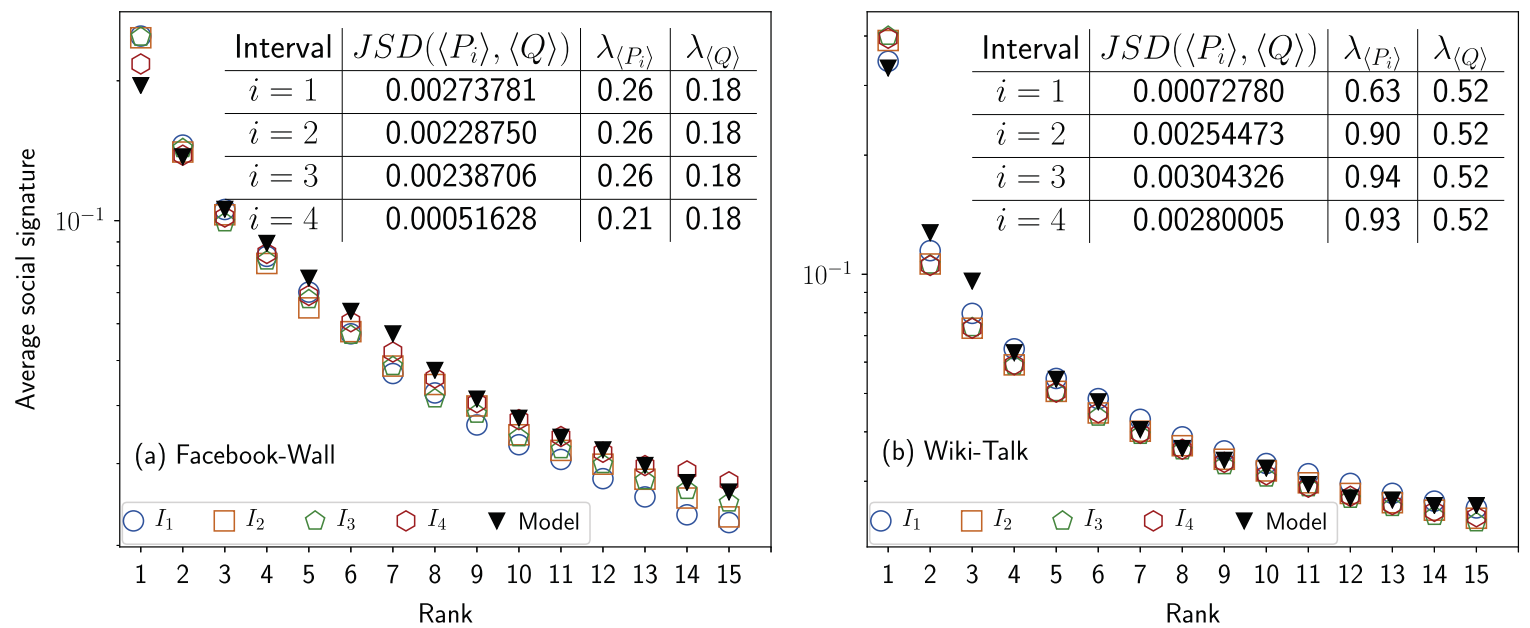

Fig. 4. Artificial signature $\langle Q\rangle$ comparing with empirical signatures $\left\langle P_{i}\right\rangle$ in 4 intervals. The two kinds of social signature show high consistence with $J S D$ value all smaller than 0.005 for both datasets, while the slope $\lambda$ of artificial signature is smaller than empirical signatures.

(i) Topological growth.

At each time step, add a new node with $m$ edges to m previously existing nodes, choosing preferentially nodes with large strength; i.e. a node $i$ is chosen according to the strength preferential probability:

$$
\Pi_{n e w \rightarrow i}=\frac{s_{i}}{\sum_{j} s_{j}},
$$

where node strength $s_{i}=\sum_{j \in \Gamma_{i}} w_{i j}$, and the sum runs over the set $\Gamma_{i}$ of neighbors of node $i$, while the sum of strength $s_{j}$ runs over all nodes in the time step. The weight of each new edge is fixed to $w_{0}=1$. Notably, each new coming node is coded by the sequential order.

(ii) Preferential interaction.

Each existing node $i$ selects $m$ other pre-existing nodes as target nodes for possible connection according to the probability:

$$
\Pi_{i \rightarrow j}=\frac{j}{\sum_{j} j}
$$

Since the nodes are added in order, a node $j$ represents the order $j$ in existing nodes, indicating the recency of the node. Accordingly, the old node tends to select relatively new nodes for interactions. Usually, interactions do not happen only once time. Node $i$ will interact with each target nodes several times. Let us say, the max number of interactions is $n$. The number of interactions is generated by uniformly distributed random numbers in the region $U[0, n]$. If two unconnected nodes are selected, the edge weight is assigned with $w_{0}=1$. If two connected nodes are selected, then their connection is strengthened; i.e. their edge weight is increased by $w_{0}=1$.

In this model, there are three parameters, the size of network $N$, the number of communication targets $m$ and the max number of interactions $n$. By setting values of these parameters, one can generate a weighted network. Then, one can infer a set of ego networks based on the weighted network. For distinguishing from the empirical results, we call ego network based on tie strength as empirical ego network, and ego network based on the preferential interaction model as artificial ego network. Correspondingly, social signatures are divided into two classes: the empirical signature $P_{i, j}$ in the interval $i$ and the artificial signature $Q_{j}$ of ego $j$. The artificial signature $Q_{j}$ is calculated by Eq. (1) and we still use the average social signature $\langle Q\rangle$ (calculated by Eq. (2)) for comparison. Notably, time interval $i$ has not been considered in the artificial signature. So, the artificial signature $Q_{j}$ and the average social signature $\langle Q\rangle$ is the special case when calculating $Q_{i, j}$ and $\left\langle Q_{i}\right\rangle$ if we set the interval $i=1$.

\subsection{The results analysis}

To get the best fitness between the artificial signature and the empirical signatures, we set a fixed network size $N=100$, and adjust the number of communication targets $m$ and the max number of interactions $n$, where $m=n=5$, for Wiki user, $m=2$ and $n=8$. Fig. 4 shows the comparisons of two kinds of signatures. In both datasets, JSD values between artificial signature and the empirical signatures are all less than 0.005 in each interval, which indicate that the generated social signature is well consistent with the empirical results. However, the slope $\lambda_{\langle Q\rangle}$ of artificial signature is smaller than the 
empirical slope $\lambda_{\left\langle P_{i}\right\rangle}$ in each dataset. The fact is that the value of slope $\lambda$ is related to the value of JSD. As is shown in subplot (a), in the interval $i=4$, the empirical slope $\lambda_{\left\langle P_{4}\right\rangle}=0.21$, which is the closest value to the slope $\lambda_{\langle Q\rangle}=0.18$ of artificial signature, while the $J S D\left(\left\langle P_{4}\right\rangle,\langle Q\rangle\right)=0.00051628$, much smaller than other intervals. In the interval $i=1$ of subplot (b), one can still find the closest $\lambda$ related with the smallest JSD. This results imply that the slope $\lambda$ is very sensitive to the shape of social signature.

Based on the results of the comparison according to the mechanisms of preferential interaction model, one can infer that: (1) the new user intend to connect the old user who has strong social tie; (2) the old user intend to communicate with new users. In FW dataset, the old user reaches to $m=5$ existing users each time, and each interaction happens randomly from 1 to $n=5$ times. In WT dataset, the old user reaches to $m=2$ existing users each time, and each interactions happens randomly from 1 to $n=8$ times.

\section{Conclusion and discussions}

In this paper, we empirically investigate collective behavior of online users' social signature by means of analyzing ego network and regenerate the heterogeneity of social signature. FBWall and Wikitalks are divided into 4 equal length time intervals. In each interval, we construct ego network in which each ego is tied at least 15 alters. Collective egos are those who appear in all 4 intervals with social signature. The statistical properties of frequency of interactions of ego network are measured by social signature $P$, persistence $J S D$, turnover $J$ and slope $\lambda$. Empirical results show that: (1) persistence of social signatures exists over time no matter what turnover of alters; (2) social signatures are heterogeneity by comparing the null model. Furthermore, we present a preferential interaction model in term of weighted network, which include two mechanisms: (1) New node follow topological growth according to the probability of nodes strength; (2) Old nodes follow preferential interaction according to the order of nodes, while in each time step, an old node reaches $m$ existing nodes and interact randomly from 1 to $n$ times. The simulation results show the high similarity of social signature regenerated by preferential interaction model with empirical results. One can find that (1) The strength of nodes causes the new coming user tend to choose the old user who has strong social tie; (2) The recency of nodes causes the old user tend to communicate with the new users. Furthermore, the heterogeneity of social signature can be adjusted by 2 parameters, the number of communicating targets $m$ and the max number of interactions $n$, for Facebook users, $m=n=5$, for Wiki user, $m=2$ and $n=8$.

Although social signatures have been found in online user's collective behaviors, there are still several issues unsolved. We only consider one possible preferential interaction, not comparing with the models such as random-walk-based model [47,48] and structural-based network model [41,42], and the properties of statistical physics still need to be deeply discussed. Besides, it also should be examined the effect of Dunbar number to social signature. Since human layers of social closeness approximately contain 5, 15, 50 and 150 individuals [27], the size of friendship may affect predictive results, while we only set the size of network as 100 . Recent, unfolding large-scale online collaborative human dynamics have been found to explain the universal double-power-law distribution [49], which may break through the limitation of our network size. Moreover, individual social attributes [50,51] may have significant influence to the way of people's communication patterns. It separates and distinguishes individuals from others and the difference of individuals have not been investigated. Finding the answers of these questions could help in deeply understanding the pattern of human social relations and behaviors.

\section{Acknowledgments}

This work is supported by the National Natural Science Foundation of China (Grant Nos. 61773248, 71771152), Research supported by The Program for Professor of Special Appointment (Eastern Scholar) at Shanghai Institutions of Higher Learning, Supported by Shuguang Program Project of Shanghai Educational Committee (Grant No. 14SG42).

\section{References}

[1] R. Lambiotte, L. Tabourier, J.C. Delvenne, Burstiness and spreading on temporal networks, Eur. Phys. J. B 86 (2013) 1.

[2] Q. Guo, W.J. Song, L. Hou, Y.L. Zhang, J.G. Liu, Effect of the time window on the heat-conduction information filtering model, Physica A 401 (2014) 15.

[3] T. Zhou, X.P. Han, X.Y. Yan, Z. Yang, Z. Zhao, B.H. Wang, Statistical mechanics on temporal and spatial activities of human, J. Univ. Electron. Sci. Tech. China 42 (2013) 481.

[4] J.G. Oliveira, A.L. Barabási, Human dynamics: Darwin and Einstein correspondence patterns, Nature 437 (2005) 1251.

[5] D. Brockman, L. Hufnagel, T. Geisel, The scaling laws of human travel, Nature 439 (2006) 462.

[6] J. Saramäki, E.A. Leicht, E. López, S.G. Roberts, F. Reed-Tsochas, R.I. Dunbar, Persistence of social signatures in human communication, Proc. Natl. Acad. Sci. USA 111 (2014) 942.

[7] A.L. Barabási, The origin of bursts and heavy tails in human dynamics, Nature 435 (2005) 207.

[8] A. Vázquez, B. Racz, A. Lukacs, A.L. Barabási, Impact of non-Poissonian activity patterns on spreading processes, Phys. Rev. Lett. 98 (2007) 158702.

[9] A. Vázquez, Exact results for the Barabási model of human dynamics, Phys. Rev. Lett. 95 (2005) 248701.

[10] A. Vázquez, J.G. Oliveira, Z. Dezsö, K.I. Goh, I. Kondor, A.L. Barabási, Modeling bursts and heavy tails in human dynamics, Phys. Rev. E 73 (2006) 036127.

[11] X.P. Han, T. Zhou, B.H. Wang, Modeling human dynamics with adaptive interest, New J. Phys. 10 (2008) 073010.

[12] M.S. Shang, G.X. Cheng, S.X. Dai, B.H. Wang, T. Zhou, Interest-driven model for human dynamics, Chin. Phys. Lett. 27 (2010) 048701.

[13] Z.D. Zhao, Z. Yang, Z. Zhang, T. Zhou, Z.G. Huang, Y.C. Lai, Emergence of scaling in human-interest dynamics, Sci. Rep. 3 (2013) 3472.

[14] J. Guo, C. Fan, Z. Guo, Weblog patterns and human dynamics with decreasing interest, Eur. Phys. J. B 81 (2011) 341. 
[15] R.D. Malmgren, D.B. Stouffer, A.E. Motter, L.A. Amaral, A Poissonian explanation for heavy tails in e-mail communication, Proc. Natl. Acad. Sci. USA 105 (2008) 18153

[16] J. Kim, D. Lee, B. Kahng, Microscopic modelling circadian and bursty pattern of human activities, PLoS One 3 (2013) e58292.

[17] S.P. Borgatti, A. Mehra, D.J. Brass, G. Labianca, Network analysis in the social sciences, Science 323 (2009) 892.

[18] R.I. Dunbar, Coevolution of neocortical size, group size and language in humans, Behav. Brain Sci. 16 (1993) 681.

[19] R.I. Dunbar, J. Bever, Neocortex size predicts group size in carnivores and some insectivores, Ethology 104 (1998) 695.

[20] R.I. Dunbar, S. Shultz, Understanding primate brain evolution, Philos. Trans. R. Soc. B 362 (2007) 649.

[21] H. Kudo, R.I. Dunbar, Neocortex size and social network size in primates, Anim. Behav. 62 (2001) 711.

[22] J. Stiller, R.I. Dunbar, Perspective-taking and memory capacity predict social network size, Soc. Netw. 29 (2007) 93.

[23] J. Powell, P.A. Lewis, N. Roberts, M. García-Fiãana, R.I. Dunbar, Orbital prefrontal cortex volume predicts social network size: An imaging study of individual differences in humans, Philos. Trans. R. Soc. B 279 (2012) 2157.

[24] H.R. Bernard, P.D. Killworth, On the social structure of an ocean-going research vessel and other important things, Soc. Sci. Res. 2 (1973) 145.

[25] G.A. Miller, The magical number seven plus or minus two: Some limits on our capacity for processing information, Psychol. Rev. 63 (1956) 81.

[26] R.I. Dunbar, Do online social media cut through the constraints that limit the size of offline social networks?, Roy. Soc. Open Sci. 3 (2016) 150292.

[27] R.I. Dunbar, V. Arnaboldi, M. Conti, A. Passarella, The structure of online social networks mirrors those in the offline world, Soc. Netw. 43 (2015) 39.

[28] R.A. Hill, R.I. Dunbar, Social network size in humans, Hum. Nat. 14 (2003) 53.

[29] R.I. Dunbar, S. Shultz, Evolution in the social brain, Science 317 (2007) 1344.

[30] T. Aledavood, E. Lõpez, S.G. Roberts, F. Reed-Tsochas, E. Moro, R.I. Dunbar, J. Saramäki, Daily rhythms in mobile telephone communication, PLoS One $10(2015)$ e 0138098

[31] Y.Y. Ahn, S. Han, H. Kwak, S. Moon, H. Jeong, Analysis of topological characteristics of huge online social networking services, in: Proceedings of the 16th International Conference on World Wide Web, ACM, 2007, pp. 835-844.

[32] S.A. Golder, D.M. Wilkinson, B.A. Huberman, Rhythms of social interaction: Messaging within a massive online network, Communities Technol. (2007) 41-66.

[33] B. Gonçalves, N. Perra, A. Vespignani, Modeling users' activity on twitter networks: Validation of dunbar's number, PLoS One 6 (2011) e22656.

[34] Q. Guo, F. Shao, Z.L. Hu, J.G. Liu, Statistical properties of the personal social network in the Facebook, Europhys. Lett. 104 (2013) 28004.

[35] J. Boase, B. Wellman, Personal relationships: On and off the internet, in: The Cambridge Handbook of Personal Relationships, 2006, p. 709.

[36] W. Youyou, M. Kosinski, D. Stillwell, Computer-based personality judgments are more accurate than those made by humans, Proc. Natl. Acad. Sci. USA 112 (2015) 1036

[37] R.M. Bond, C.J. Fariss, J.J. Jones, A.D. Kramer, C. Marlow, J.E. Settle, J.H. Fowler, A 61-million-person experiment in social influence and political mobilization, Nature 489 (2012) 295.

[38] S. Aral, D. Walker, Tie strength, embeddedness, and social influence: A large-scale networked experiment, Manage. Sci. 60 (2014) 1352.

[39] L. Backstrom, J. Kleinberg, Romantic Partnerships and the Dispersion of Social Ties: A Network Analysis of Relationship Status on Facebook, ACM, 2014, p. 831.

[40] D. Easley, J. Kleinberg, Networks, Crowds, and Markets: Reasoning About a Highly Connected World, Cambridge University Press, 2010.

[41] W.X. Wang, B. Hu, T. Zhou, B.H. Wang, Y.B. Xie, Mutual selection model for weighted networks, Phys. Rev. E 72 (2005) 046140.

[42] Z. Pan, X. Li, X. Wang, Generalized local-world models for weighted networks, Phys. Rev. E 73 (2006) 056109.

[43] J.G. Liu, Z.M. Ren, Q. Guo, D.B. Chen, Evolution characteristics of the network core in the Facebook, PLoS One 9 (2014) e104028.

[44] B. Viswanath, A. Mislove, M. Cha, K.P. Gummadi, On the evolution of user interaction in facebook, in: Proceedings of the 2nd ACM Workshop on Online Social Networks, 2009, pp. 37-42.

[45] A. Paranjape, A.R. Benson, J. Leskovec, Motifs in temporal networks, in: Proceedings of the Tenth ACM International Conference on Web Search and Data Mining, 2017.

[46] J. Leskovec, D.P. Huttenlocher, J.M. Kleinberg, Governance in social media: A case study of the Wikipedia promotion process, in: ICWSM, 2010

[47] M. Starnini, A. Baronchelli, R. Pastor-Satorras, Model reproduces individual, group and collective dynamics of human contact networks, Soc. Netw. 47 (2016) 130.

[48] T. Jia, D. Wang, B.K. Szymanski, Quantifying patterns of research-interest evolution, Nat. Hum. Behav. 1 (2017) 0078.

[49] Y. Zha, T. Zhou, C. Zhou, Unfolding large-scale online collaborative human dynamics, Proc. Natl. Acad. Sci. USA 113 (2016) 14627.

[50] S. Centellegher, E. Lápez, J. Saramäki, B. Lepri, Personality traits and ego-network dynamics, PLoS One 12 (2017) e0173110.

[51] H.H. Jo, R.I. Dunbar, J. Saramäki, K. Kaski, Dynamics of close relationships for the life-course migration, Sci. Rep. 4 (2014) 6988. 